\title{
On the initial value problem for systems of linear differential equations with argument deviations
}

\author{
Andrei Rontó
}




\title{
ON THE INITIAL VALUE PROBLEM FOR SYSTEMS OF LINEAR DIFFERENTIAL EQUATIONS WITH ARGUMENT DEVIATIONS
}

\author{
ANDREI RONTÓ
}

[Received: 25 February, 2005]

\begin{abstract}
Aвstract. We establish new efficient conditions sufficient for the unique solvability of the Cauchy problem for many-dimensional systems of linear differential equations with arbitrary argument deviations.
\end{abstract}

Mathematics Subject Classification: 34K06, 34K10

Keywords: Functional differential equation, Cauchy problem, initial value problem, equation with deviated argument

\section{InTRODUCTION}

In the class of functional differential equations, the question on the solvability of the initial value problem, as is well-known, is non-trivial even in the linear case. For example, the simplest scalar functional differential equation

$$
u^{\prime}(t)=\frac{u(b)}{b}+q(t), \quad t \in[0, b],
$$

has no solutions satisfying the initial condition

$$
u(0)=c,
$$

provided that the function $q \in L_{1}([0, b], \mathbb{R})$ and the real constant $c$ are related by the inequality

$$
\int_{0}^{b} q(s) d s \neq-c .
$$

From the standpoint of the theory of ordinary differential equations, the fact indicated may seem somewhat strange if we note that (1.1) is a scalar linear equation with the constant coefficient $b^{-1}$, and this coefficient, moreover, becomes arbitrarily small when the length of the interval, $b$, increases to $+\infty$. Such phenomena are, however, not uncommon, if not typical, for functional differential equations.

Supported in part by AS CR, Institutional Research Plan No. AV0Z10190503, and by the President of Ukraine through Grant No. F8/331/2005. 
At present, there are not but a few sharp and efficient conditions guaranteeing the unique solvability of the initial value problem for differential equations with arbitrary argument deviations, and most of them are available for the one-dimensional case only (see, e. g., [4] and references therein).

In this paper, we continue the study initiated in $[2,3,6]$ and establish some new conditions guaranteeing the unique solvability of the problem

$$
\begin{gathered}
u_{k}^{\prime}(t)=\sum_{j=1}^{n} r_{k j}(t) u_{j}\left(\omega_{k j}(t)\right)+q_{k}(t), \quad t \in[a, b], k=1,2, \ldots, n, \\
u_{k}(\tau)=c_{k}, \quad k=1,2, \ldots, n .
\end{gathered}
$$

Here, $n \in \mathbb{N}, \tau$ is a given point from $[a, b], q_{k}:[a, b] \rightarrow \mathbb{R}$ and $r_{k j}:[a, b] \rightarrow \mathbb{R}$, $k, j=1,2, \ldots, n$, are Lebesgue integrable functions, $\left\{c_{k} \mid k=1,2, \ldots, n\right\} \subset \mathbb{R}$ are given constants, and $\omega_{k j}, k, j=1,2, \ldots, n$, are arbitrary measurable transformations of the interval $[a, b]$ to itself.

By a solution of problem (1.2), (1.3), as usual [1], we mean a vector-function $u=\left(u_{k}\right)_{k=1}^{n}:[a, b] \rightarrow \mathbb{R}^{n}$ with absolutely continuous components $u_{k}, k=1,2, \ldots, n$, satisfying relations (1.2) almost everywhere on $[a, b]$ and possessing properties (1.3).

\section{Notation}

The following notation is used throughout the paper.

(1) $\mathbb{N}=\{1,2, \ldots\},, \mathbb{R}=(-\infty, \infty)$.

(2) $L_{1}\left([a, b], \mathbb{R}^{n}\right)$ is the Banach space of Bochner integrable functions $u:[a, b] \rightarrow$ $\mathbb{R}^{n}$ endowed by the norm

$$
L_{1}\left([a, b], \mathbb{R}^{n}\right) \ni u=\left(u_{k}\right)_{k=1}^{n} \longmapsto \max _{k=1,2, \ldots, n} \int_{a}^{b}\left|u_{k}(t)\right| d t .
$$

(3) $C\left([a, b], \mathbb{R}^{n}\right)$ is the Banach space of continuous functions $u:[a, b] \rightarrow \mathbb{R}^{n}$ endowed by the norm

$$
C\left([a, b], \mathbb{R}^{n}\right) \ni u=\left(u_{k}\right)_{k=1}^{n} \longmapsto \max _{k=1,2, \ldots, n} \max _{t \in[a, b]}\left|u_{k}(t)\right| .
$$

(4) $C_{\tau}\left([a, b], \mathbb{R}^{n}\right)$, where $\tau \in[a, b]$, is the subspace of $C\left([a, b], \mathbb{R}^{n}\right)$ constituted by those functions $u$ for which $u(\tau)=0$.

(5) $r(A)$ is the spectral radius of a bounded linear operator $A$.

(6) $\mathrm{GL}_{n}(\mathbb{R})$ is the algebra of the $n$-dimensional square matrices with real elements.

\section{Conditions For the UNiQue SOlvabiLITY OF PROBLEM (1.2), (1.3)}

In this section, we present our main results concerning the initial value problem (1.2), (1.3). The proofs are given later, in Section 5. 
3.1. Existence of Green's operator. The following statement provides conditions sufficient for the unique solvability of the initial value problem (1.2), (1.3).

Theorem 3.1. Let there exist some $\alpha \in[1,+\infty)$ and $\left\{\gamma_{k} \mid k=1,2, \ldots, n\right\} \subset$ $(0,+\infty)$ such that one of the following conditions is satisfied:

$$
\begin{gathered}
\max _{k=1,2, \ldots, n} \operatorname{vrai}_{t \in[a, b] \backslash\{\tau\}} \frac{1}{\gamma_{k}|t-\tau|^{\alpha-1}} \sum_{j=1}^{n} \gamma_{j}\left|r_{k j}(t)\right|\left|\omega_{k j}(t)-\tau\right|^{\alpha}<\alpha, \\
\max _{k=1,2, \ldots, n} \sup _{t \in[a, b] \backslash\{\tau\}} \frac{\operatorname{sign}(t-\tau)}{\gamma_{k}|t-\tau|^{\alpha}} \sum_{j=1}^{n} \gamma_{j} \int_{\tau}^{t}\left|r_{k j}(s)\right|\left|\omega_{k j}(s)-\tau\right|^{\alpha} d s<1, \\
\max _{k=1,2, \ldots, n} \operatorname{vrai}_{t \in[a, b] \backslash\{\tau\}} \sum_{l=1}^{n} \frac{\left|r_{k l}(t)\right|}{\gamma_{k}|t-\tau|^{\alpha-1}} \sum_{j=1}^{n} \gamma_{j}\left|\int_{\tau}^{\omega_{k l}(t)}\right| r_{l j}(s)|| \omega_{l j}(s)-\left.\tau\right|^{\alpha} d s \mid<\alpha .
\end{gathered}
$$

Then the initial value problem (1.2), (1.3) is uniquely solvable for arbitrary constants $\left\{c_{k} \mid k=1,2, \ldots, n\right\} \subset \mathbb{R}$ and integrable functions $q_{k}:[a, b] \rightarrow \mathbb{R}, k=$ $1,2, \ldots, n$.

None of the strict inequalities (3.1a), (3.1b), and (3.1c) assumed in Theorem 3.1 can be replaced by the corresponding non-strict inequality. For instance, the condition

$$
\max _{k=1,2, \ldots, n} \underset{v \operatorname{vrai} \max }{\max \backslash \backslash\{\tau\}} \frac{1}{\gamma_{k}|t-\tau|^{\alpha-1}} \sum_{j=1}^{n} \gamma_{j}\left|r_{k j}(t)\right|\left|\omega_{k j}(t)-\tau\right|^{\alpha} \leq \alpha
$$

does not guarantee the unique solvability of problem (1.2), (1.3) because, as follows from the example given below, for any $\alpha \in[1,+\infty)$ and $\tau \in[a, b]$, one can specify a functional differential system (1.2) for which relation (3.2) is satisfied but the Cauchy problem (1.3) is not uniquely solvable.

Example. Given $\tau \in[a, b]$ and $\alpha \in[1,+\infty)$, consider the scalar functional differential equation

$$
u^{\prime}(t)=\frac{\alpha|t-\tau|^{\alpha-1}}{|\theta-\tau|^{\alpha}} \operatorname{sign}(t-\tau) u(\theta), \quad t \in[a, b],
$$

where $\theta \in[a, b]$ is an arbitrary point different from $\tau$. This equation, clearly, has form form (1.2) with $n=1, q_{1}(t)=0, \omega_{11}(t)=\theta$, and

$$
r_{11}(t)=\frac{\alpha|t-\tau|^{\alpha-1}}{|\theta-\tau|^{\alpha}} \operatorname{sign}(t-\tau)
$$

for almost every $t \in[a, b]$. It is easy to verify that, for an arbitrary $\lambda$, the function

$$
u(t)=\lambda|t-\tau|^{\alpha}, \quad t \in[a, b],
$$

is a solution of the homogeneous Cauchy problem

$$
u(\tau)=0
$$


for equation (3.3). However, condition (3.2) is satisfied for this equation because

$$
\underset{t \in[a, b] \backslash\{\tau\}}{\operatorname{vraim} \max } \frac{1}{\gamma_{1}|t-\tau|^{\alpha-1}} \gamma_{1}\left|r_{11}(t)\right|\left|\omega_{11}(t)-\tau\right|^{\alpha}=\alpha .
$$

One can verify that the non-strict versions of inequalities (3.1b) and (3.1c) are also satisfied in this case.

3.2. Positivity of Green's operator. The conditions given below guarantee not only existence but also the positivity of Green's operator associated with the homogeneous Cauchy problem (1.2), (1.3).

Theorem 3.2. Let there exist some $\left\{\sigma_{1}, \sigma_{2}, \ldots, \sigma_{n}\right\} \subset\{-1,1\}$ such that

$$
\sigma_{k} \sigma_{j} r_{k j}(t) \operatorname{sign}(t-\tau) \geq 0
$$

for almost every $t \in[a, b]$ and all $k, j=1,2, \ldots, n$. Moreover, assume the existence of some constants $\alpha \in[1,+\infty)$ and $\left\{\gamma_{k} \mid k=1,2, \ldots, n\right\} \subset(0,+\infty)$ such that one of the following conditions is satisfied for all $k=1,2, \ldots, n:^{*}$

$$
\begin{aligned}
& \underset{t \in[a, b] \backslash\{\tau\}}{\operatorname{vrai} \max } \frac{\sigma_{k} \operatorname{sign}(t-\tau)}{\gamma_{k}|t-\tau|^{\alpha-1}} \sum_{j=1}^{n} \sigma_{j} \gamma_{j} r_{k j}(t)\left|\omega_{k j}(t)-\tau\right|^{\alpha}<\alpha, \\
& \sup _{t \in[a, b] \backslash\{\tau\}} \frac{\sigma_{k}}{\gamma_{k}|t-\tau|^{\alpha}} \sum_{j=1}^{n} \sigma_{j} \gamma_{j} \int_{\tau}^{t} r_{k j}(s)\left|\omega_{k j}(s)-\tau\right|^{\alpha} d s<1, \\
& \underset{t \in[a, b] \backslash\{\tau\}}{\operatorname{vraimax}} \frac{\sigma_{k} \operatorname{sign}(t-\tau)}{\gamma_{k}|t-\tau|^{\alpha-1}} \sum_{j=1}^{n} r_{k j}(t) \sum_{l=1}^{n} \sigma_{l} \gamma_{l} \int_{\tau}^{\omega_{k j}(t)} r_{j l}(s)\left|\omega_{j l}(s)-\tau\right|^{\alpha} d s<\alpha .
\end{aligned}
$$

Then the initial value problem (1.2), (1.3) is uniquely solvable for arbitrary constants $\left\{c_{k} \mid k=1,2, \ldots, n\right\} \subset \mathbb{R}$ and integrable functions $q_{k}:[a, b] \rightarrow \mathbb{R}, k=$ $1,2, \ldots, n$. If, in addition, $q_{k}$ and $c_{k}, k=1,2, \ldots, n$, possess the property

$$
\sigma_{k}\left(\int_{\tau}^{t} q_{k}(s) d s+c_{k}\right) \geq 0, \quad t \in[a, b], k=1,2, \ldots, n,
$$

then the unique solution $u=\left(u_{k}\right)_{k=1}^{n}$ of problem (1.2), (1.3) satisfies the condition

$$
\sigma_{k} u_{k}(t) \geq 0, \quad t \in[a, b], k=1,2, \ldots, n .
$$

The example presented in Section 3.1 shows that conditions (3.5a), (3.5b), and (3.5c) are optimal in the sense that none of them can be weakened to the corresponding non-strict inequality.

\section{General statements}

The theorems presented above rely upon some general results on linear integral functional operators. The results mentioned are presented in this section.

${ }^{*}$ We emphasise that one and the same condition should be satisfied for all $k$ from 1 to $n$. 
4.1. Spectra of linear integral operators with inner superpositions. Let $R_{i}$ : $[a, b] \rightarrow \mathrm{GL}_{n}(\mathbb{R}), i=1,2, \ldots, N$, where $N \in \mathbb{N}$, be matrix-valued functions with Lebesgue integrable components, and $\omega_{i}:[a, b] \rightarrow[a, b], i=1,2, \ldots, N$, be measurable functions.

For every $u$ from $C_{\tau}\left([a, b], \mathbb{R}^{n}\right),{ }^{\dagger}$ we put

$$
(B u)(t):=\sum_{i=1}^{N} \int_{\tau}^{t} R_{i}(s) u\left(\omega_{i}(s)\right) d s, \quad t \in[a, b] .
$$

Obviously, $B$ is a bounded linear mapping of the space $C_{\tau}\left([a, b], \mathbb{R}^{n}\right)$ to itself. One can show that operator (4.1) is completely continuous in the space mentioned.

Until the end of this section, we fix a diagonal matrix

$$
\Sigma=\operatorname{diag}\left\{\sigma_{1}, \sigma_{2}, \ldots, \sigma_{n}\right\}
$$

with components $\left\{\sigma_{1}, \sigma_{2}, \ldots, \sigma_{n}\right\} \subset\{-1,1\}$. For the sake of brevity we introduce a definition.

Definition 4.1. For $\{x, y\} \subset \mathbb{R}^{n}$ we write $x \geq_{\Sigma} y$ (resp., $x>_{\Sigma} y$ ) if, and only if $\sigma_{k} x_{k} \geq \sigma_{k} y_{k}$ (resp., $\sigma_{k} x_{k}>\sigma_{k} y_{k}$ ) for all $k=1,2, \ldots, n$.

The following statement provides a convenient way to estimate the spectral radius of operator (4.1).

Proposition 4.1. Let each of the functions $R_{v}:[a, b] \rightarrow \mathrm{GL}_{n}(\mathbb{R}), v=0,1, \ldots, N$, satisfy the condition

$$
\Sigma R_{v}(t) \Sigma \operatorname{sign}(t-\tau) \geq 0 \quad \text { for a. e. } t \in[a, b]
$$

with respect to the given matrix (4.2). Furthermore, assume that there exists an absolutely continuous function $y:[a, b] \rightarrow \mathbb{R}^{n}$ possessing the properties

$$
y(\tau)=0 ; \quad y(t)>_{\Sigma} 0 \text { for all } t \in[a, b] \backslash\{\tau\}
$$

and

$$
y^{\prime}(t) \operatorname{sign}(t-\tau) \geq_{\Sigma} 0 \quad \text { for a. e. } t \in[a, b],
$$

and such that, with a certain constant $\varrho \in(1,+\infty)$, the integral relation

$$
y(t) \geq_{\Sigma} \varrho \sum_{i=1}^{N} \int_{\tau}^{t} R_{i}(s) y\left(\omega_{i}(s)\right) d s
$$

is true for every $t \in[a, b]$.

Then the spectral radius $r(B)$ of the operator $B: C_{\tau}\left([a, b], \mathbb{R}^{n}\right) \rightarrow C_{\tau}\left([a, b], \mathbb{R}^{n}\right)$ given by formula (4.1) is less than 1.

Remark 4.1. The inequality in (4.3) and similar relations below is understood componentwise.

\footnotetext{
${ }^{\dagger}$ See notation (4), Section 2.
} 
Proposition 4.1 is obtained from [3, Corollary 6] and the argument given in the proof of [6, Theorem 1]. The details are omitted here.

Proposition 4.2. Let the functions $R_{v}:[a, b] \rightarrow \mathrm{GL}_{n}(\mathbb{R}), v=0,1, \ldots, N$, satisfy condition (4.3). Moreover, there exist some constants $\gamma \in[0,1)$ and $\alpha \in[1,+\infty)$ and an $n$-dimensional vector $g$ with the property

$$
g>_{\Sigma} 0
$$

such that

$$
\sum_{v=1}^{N} \int_{\tau}^{t} R_{v}(s) g\left|\omega_{v}(s)-\tau\right|^{\alpha} d s \leq_{\Sigma} \gamma|t-\tau|^{\alpha} g, \quad t \in[a, b] .
$$

Then the spectral radius $r(B)$ of operator (4.1) in the space $C_{\tau}\left([a, b], \mathbb{R}^{n}\right)$ is less than 1.

Proof. Let $y:[a, b] \rightarrow \mathbb{R}^{n}$ be the function given by the formula

$$
y(t):=|t-\tau|^{\alpha} g, \quad t \in[a, b],
$$

where $\alpha$ and $g$ are the values appearing in the formulation of Theorem 4.2. Function (4.9) obviously possesses properties (4.4). One can verify that, for almost every $t \in[a, b]$,

$$
y^{\prime}(t)=\alpha|t-\tau|^{\alpha-1} \operatorname{sign}(t-\tau) g,
$$

and, hence, function (4.9) also satisfies condition (4.5). Furthermore, it follows from assumption (4.8) that function (4.9) satisfies condition (4.6) with $\varrho:=\gamma^{-1}$. Therefore, it remains to apply Proposition 4.1.

Proposition 4.3. Let the functions $R_{v}:[a, b] \rightarrow \mathrm{GL}_{n}(\mathbb{R}), v=0,1, \ldots, N$, satisfy condition (4.3). Let, moreover, there exist some constants $\gamma \in[0,1)$ and $\alpha \in[1,+\infty)$ and n-dimensional vector $g$ with property (4.7) such that the following relation is satisfied for almost every $t$ from $[a, b]$ :

$$
\sum_{v=1}^{N} R_{v}(t) \sum_{i=1}^{N} \int_{\tau}^{\omega_{v}(t)} R_{i}(s) g\left|\omega_{i}(s)-\tau\right|^{\alpha} d s \cdot \operatorname{sign}(t-\tau) \leq_{\Sigma} \gamma \alpha|t-\tau|^{\alpha-1} g .
$$

Then the spectral radius $r(B)$ of operator $(4.1)$ in the space $C_{\tau}\left([a, b], \mathbb{R}^{n}\right)$ is less than 1 .

To establish the theorem formulated above, one should combine Theorem 4.1, [3, Corollary 7], and [3, Remark 6].

${ }^{\ddagger}$ It is sufficient to consider the case where $\gamma>0$ because if $\gamma=0$, then the corresponding relation (4.6) is satisfied with an arbitrary $\varrho \in(1,+\infty)$. 
Proposition 4.4. Assume that there exists a certain diagonal matrix (4.2) with $\left\{\sigma_{1}, \sigma_{2}, \ldots, \sigma_{n}\right\} \subset\{-1,1\}$ for which the functions $R_{v}:[a, b] \rightarrow \mathrm{GL}_{n}(\mathbb{R}), v=$ $0,1, \ldots, N$, satisfy condition (4.3) and, moreover, let there exist some constants $\gamma \in$ $[0,1)$ and $\alpha \in[1,+\infty)$ and $n$-dimensional vector $g$ with property (4.7) such that

$$
\sum_{v=1}^{N} R_{v}(t) g\left|\omega_{v}(t)-\tau\right|^{\alpha} \operatorname{sign}(t-\tau) \leq_{\Sigma} \gamma \alpha|t-\tau|^{\alpha-1} g \quad \text { for a. e. } t \in[a, b] .
$$

Then the spectral radius $r(B)$ of operator $(4.1)$ in the space $C_{\tau}\left([a, b], \mathbb{R}^{n}\right)$ is less than 1.

Similarly to Proposition 4.3, the assertion of Proposition 4.4 is deduced from [3, Corollary 8] and [3, Remark 6].

4.2. Solvability of linear initial value problems. The combination of the statements presented in Section 4.1 with Lemma 4.1 given below leads one to efficient conditions of solvability of the Cauchy problem (1.3),

$$
u(\tau)=c,
$$

for the functional differential equation of the form

$$
u^{\prime}(t)=\sum_{i=1}^{N} R_{i}(t) u\left(\omega_{i}(t)\right)+q(t), \quad t \in[a, b],
$$

where $N \in \mathbb{N}$, the functions $R_{i}:[a, b] \rightarrow \mathrm{GL}_{n}(\mathbb{R}), i=1,2, \ldots, N$, and $q:[a, b] \rightarrow$ $\mathbb{R}^{n}$ are integrable, and $\omega_{i}:[a, b] \rightarrow[a, b], i=1,2, \ldots, N$, are measurable.

Lemma 4.1. If an absolutely continuous function $u:[a, b] \rightarrow \mathbb{R}^{n}$ is a solution of the Cauchy problem (4.13), (4.12), then it satisfies the equation

$$
u(t)=(B u)(t)+c+\int_{\tau}^{t} q(s) d s, \quad t \in[a, b] .
$$

Conversely, every continuous solution $u(\cdot)$ of equation (4.14) is also a solution of problem (4.12), (4.13)

The assertion of Lemma 4.1, which is easily verified by direct computation, allows one to interpret the space of solutions of the homogeneous problem

$$
\begin{gathered}
u^{\prime}(t)=\sum_{i=1}^{N} R_{i}(t) u\left(\omega_{i}(t)\right), \quad t \in[a, b], \\
u(\tau)=0,
\end{gathered}
$$

as the set of fixed points of operator (4.1) considered in the space $C_{\tau}\left([a, b], \mathbb{R}^{n}\right)$. 
Lemma 4.2. Let the functions $R_{i}:[a, b] \rightarrow \mathrm{GL}_{n}(\mathbb{R}), i=1,2, \ldots, N$, satisfy condition (4.3) for some matrix (4.2) with elements $\left\{\sigma_{1}, \sigma_{2}, \ldots, \sigma_{n}\right\} \subset\{-1,1\}$. Then

$$
\sum_{i=1}^{N} R_{i}(t) u\left(\omega_{i}(t)\right) \operatorname{sign}(t-\tau) \geq_{\Sigma} 0 \quad \text { for a. e. } t \in[a, b],
$$

provided that the continuous function $u:[a, b] \rightarrow \mathbb{R}^{n}$ satisfies the condition

$$
u(t) \geq_{\Sigma} 0, \quad t \in[a, b] .
$$

Proof. Indeed, let $u:[a, b] \rightarrow \mathbb{R}^{n}$ be an arbitrary continuous function possessing property (4.16), or, which is the same (see Definition 4.1), such that the componentwise inequality

$$
\Sigma u(t) \geq 0, \quad t \in[a, b],
$$

is true. Since $\left\{\sigma_{k} \mid k=1,2, \ldots, n\right\} \subset\{-1,1\}$, it is obvious that $\Sigma^{2}$ is the identity matrix and, hence, according to Definition 4.1, relation (4.15) can be rewritten in the form

$$
\Sigma \sum_{i=1}^{N} R_{i}(t) \Sigma \Sigma u\left(\omega_{i}(t)\right) \operatorname{sign}(t-\tau) \geq 0 \quad \text { for a. e. } t \in[a, b] .
$$

In view of (4.17), we see that $\Sigma u\left(\omega_{i}(t)\right) \geq 0$ for every $i=1,2, \ldots, N$ and almost every $t \in[a, b]$. Therefore, (4.18) is true provided that each of the functions $R_{i}:[a, b] \rightarrow$ $\mathrm{GL}_{n}(\mathbb{R}), i=1,2, \ldots, N$, satisfies condition (4.3).

Lemma 4.3. Condition (4.3) is satisfied if, and only if

$$
\sigma_{k} \sigma_{j} r_{k j}^{(v)}(t) \operatorname{sign}(t-\tau) \geq 0
$$

for almost every $t \in[a, b]$ and all $k, j=1,2, \ldots, n$ and $v=1,2, \ldots, N$, where $r_{k j}^{(v)}$ stand for the corresponding elements of the matrix $R_{v}=\left(r_{k j}^{(v)}\right)_{k, j=1}^{n}$.

Proor. It is not difficult to verify that, for all $v=1,2, \ldots, N$ and almost every $t \in[a, b]$, the matrix $\Sigma R_{v}(t) \Sigma$ in the left-hand side of (4.3) has the form

$$
\Sigma R_{v}(t) \Sigma=\left(\begin{array}{cccc}
r_{11}^{(v)}(t) & \sigma_{1} \sigma_{2} r_{11}^{(v)}(t) & \ldots & \sigma_{1} \sigma_{n} r_{1 n}^{(v)}(t) \\
\sigma_{2} \sigma_{1} r_{21}^{(v)}(t) & r_{11}^{(v)}(t) & \ldots & \sigma_{2} \sigma_{n} r_{2 n 1}^{(v)}(t) \\
\ldots & \ldots & \ldots & \ldots \\
\sigma_{n} \sigma_{1} r_{n 1}^{(v)}(t) & \sigma_{n} \sigma_{2} r_{n 2}^{(v)}(t) & \ldots & r_{n n}^{(v)}(t)
\end{array}\right),
$$

and, therefore, (4.3) means nothing but the fulfilment of (4.19) for almost every $t \in$ $[a, b]$ and all $k$ and $j$ from 1 to $n$.

The following statement summarises the conditions established by the propositions above. 
Proposition 4.5. Assume that the matrix-valued functions $R_{v}:[a, b] \rightarrow \mathrm{GL}_{n}(\mathbb{R})$, $v=0,1, \ldots, N$, satisfy condition (4.3) with respect to some matrix (4.2) with components $\left\{\sigma_{1}, \sigma_{2}, \ldots, \sigma_{n}\right\} \subset\{-1,1\}$. Moreover, let there exist some constants $\gamma \in[0,1)$ and $\alpha \in[1,+\infty)$ and vector $g \in \mathbb{R}^{n}$ with property (4.7) such that one of conditions (4.8), (4.10), and (4.11) is true.

Then the spectral radius of operator (4.1) in $C_{\tau}\left([a, b], \mathbb{R}^{n}\right)$ admits the estimate

$$
r(B)<1 .
$$

Proof. One should apply Proposition 4.2, 4.3, or 4.4, depending on the conditions assumed.

Combining Proposition 4.5 with with Lemma 4.1, we arrive at

Theorem 4.1. Under conditions of Proposition 4.5, the initial value problem (4.12), (4.13) has a unique solution $u(\cdot)$ for any $q \in L_{1}\left([a, b], \mathbb{R}^{n}\right)$ and $c \in \mathbb{R}^{n}$. Moreover, if $q$ and $c$ possess the property

$$
\int_{\tau}^{t} q(s) d s \geq_{\Sigma}-c, \quad t \in[a, b]
$$

then the solution $u(\cdot)$ of problem (4.12), (4.13) satisfies condition (4.16).

Proof. The validity of the first assertion of the theorem is a consequence of Proposition 4.5 and Lemma 4.1. The second assertion follows from Lemma 4.2.

For systems (4.13) with coefficients not satisfying conditions of type (4.3), the following somewhat weaker statement is true.

Theorem 4.2. Assume that, for some constants $\gamma \in[0,1)$ and $\alpha \in[1,+\infty)$ and vector $g=\left(g_{k}\right)_{k=1}^{n} \in \mathbb{R}^{n}$ with strictly positive components, one of the following conditions is satisfied: ${ }^{\S}$

$$
\begin{gathered}
\sum_{v=1}^{N} \int_{\tau}^{t}\left|R_{v}(s)\right| g\left|\omega_{v}(s)-\tau\right|^{\alpha} d s \cdot \operatorname{sign}(t-\tau) \leq \gamma|t-\tau|^{\alpha} g, \quad t \in[a, b], \\
\sum_{v=1}^{N} \operatorname{sign}\left(\omega_{v}(t)-\tau\right)\left|R_{v}(t)\right| \sum_{i=1}^{N} \int_{\tau}^{\omega_{v}(t)}\left|R_{i}(s)\right| g\left|\omega_{i}(s)-\tau\right|^{\alpha} d s \\
\leq \gamma \alpha|t-\tau|^{\alpha-1} g \text { for a. e. } t \in[a, b],
\end{gathered}
$$

and

$$
\sum_{v=1}^{N}\left|R_{v}(t)\right| g\left|\omega_{v}(t)-\tau\right|^{\alpha} \leq \gamma \alpha|t-\tau|^{\alpha-1} g \quad \text { for a. e. } t \in[a, b] .
$$

${ }^{\S}$ In (4.21) and similar relations below, we use the notation $|M|=\left(\left|m_{k j}\right|\right)_{k, j=1}$ for a matrix $M=$ $\left(m_{k j}\right)_{k, j=1}$. 
Then the initial value problem (4.12), (4.13) has a unique solution $u(\cdot)$ for any $q \in L_{1}\left([a, b], \mathbb{R}^{n}\right)$ and $c \in \mathbb{R}^{n}$.

It should be noted that, in contrast to Theorem 4.1, the solution $u$ of problem (4.12), (4.13) may not possess property (4.16) under assumptions of Theorem 4.2 even if $q$ and $c$ satisfy condition (4.20).

4.3. Auxiliary statements and the proof of Theorem 4.2. We need the following lemma.

Lemma 4.4. Let $A: C_{\tau}\left([a, b], \mathbb{R}^{n}\right) \rightarrow C_{\tau}\left([a, b], \mathbb{R}^{n}\right)$ be the operator defined by the formula

$$
(A u)(t):=\sum_{i=1}^{N} \int_{\tau}^{t}\left|R_{i}(s)\right| \operatorname{sign}(s-\tau) u\left(\omega_{i}(s)\right) d s, \quad t \in[a, b] .
$$

Then the componentwise estimate

$$
-(A u)(t) \leq(B u)(t) \leq(A u)(t), \quad t \in[a, b],
$$

is true for an arbitrary continuous function $u:[a, b] \rightarrow \mathbb{R}^{n}$ possessing the properties

$$
u(\tau)=0 ; \quad u(t) \geq 0, \quad t \in[a, b] .
$$

Recall that the operator $B: C_{\tau}\left([a, b], \mathbb{R}^{n}\right) \rightarrow C_{\tau}\left([a, b], \mathbb{R}^{n}\right)$ is determined by the matrix-valued functions $R_{i}:[a, b] \rightarrow \mathrm{GL}_{n}(\mathbb{R}), i=1,2, \ldots, N$, according to formula (4.1).

Proof of Lemma 4.4. Indeed, let $u$ satisfy (4.26). Then, according to (4.1), we have

$$
-\sum_{i=1}^{N} \int_{\tau}^{t}\left|R_{i}(s)\right| u\left(\omega_{i}(s)\right) d s \leq(B u)(t) \leq \sum_{i=1}^{N} \int_{\tau}^{t}\left|R_{i}(s)\right| u\left(\omega_{i}(s)\right) d s
$$

if $t \geq \tau$, and, similarly,

$$
\sum_{i=1}^{N} \int_{\tau}^{t}\left|R_{i}(s)\right| u\left(\omega_{i}(s)\right) d s \leq(B u)(t) \leq-\sum_{i=1}^{N} \int_{\tau}^{t}\left|R_{i}(s)\right| u\left(\omega_{i}(s)\right) d s
$$

if $t \leq \tau$. This, in view of (4.24), leads us to (4.25).

The following statement is, in fact, Theorem 5.3 from [5].

Theorem 4.3. Let $K$ be a normal and reproducing cone in a real Banach space $X$ and $A$ and $B$ be linear operators in $X$ such that

$$
\{A u+B u, A u-B u\} \subseteq K \quad \text { for an arbitrary } u \in K .
$$

Then the relation

$$
r(B) \leq r(A)
$$

is true for the spectral radii of $A$ and $B$. 
Remark 4.2. Theorem 5.3 from [5] contains the assumption that the mapping $A$ should leave the cone $K$ invariant, i. e.,

$$
A(K) \subseteq K \text {. }
$$

It easy to see that inclusion (4.29) follows immediately from condition (4.27) and, thus, can be omitted in the formulation.

Now we are in position to prove Theorem 4.2.

Proof of Theorem 4.2. Let us set

$$
X:=C_{\tau}\left([a, b], \mathbb{R}^{n}\right)
$$

and

$$
\begin{array}{r}
K:=\left\{u=\left(u_{k}\right)_{k=1}^{n} \in C_{\tau}\left([a, b], \mathbb{R}^{n}\right) \mid u_{k}(t) \geq 0 \text { for all } k=1,2, \ldots, n\right. \\
\text { and } t \in[a, b]\} .
\end{array}
$$

Obviously, set (4.30) (see notation (4), Section 2) is a Banach space with respect to the uniform norm (2.1). It is also easy to verify that set (4.31) is a cone in $X$ and, moreover, this cone is normal and reproducing (see, e. g., [5], $\$ 3.2$ and 3.3, for the terminology).

It follows from Lemma 4.4 that relation (4.25) holds for the operators $A$ and $B$ given by formulae (4.1) and (4.24), respectively. Taking (4.31) into account, we conclude that the operators mentioned have property (4.27), and therefore Theorem 4.3 ensures the validity of estimate (4.28).

Relations (4.21) and (4.22), as is easy to see, can be rewritten as

$$
\sum_{v=1}^{N} \int_{\tau}^{t}\left|R_{v}(s)\right| \operatorname{sign}(s-\tau) g\left|\omega_{v}(s)-\tau\right|^{\alpha} d s \leq \gamma|t-\tau|^{\alpha} g, \quad t \in[a, b],
$$

and

$$
\begin{aligned}
\sum_{v=1}^{N}\left|R_{v}(t)\right| \sum_{i=1}^{N} \int_{\tau}^{\omega_{v}(t)}\left|R_{i}(s)\right| \operatorname{sign}(s-\tau) g \mid \omega_{i}(s) & -\left.\tau\right|^{\alpha} d s \leq \\
& \leq \gamma \alpha|t-\tau|^{\alpha-1} g, \quad t \in[a, b],
\end{aligned}
$$

respectively. Therefore, assumptions (4.21), (4.22), and (4.23) guarantee the fulfilment of the corresponding relations (4.8), (4.10), and (4.11) with $\Sigma$ equal to the identity matrix and $R_{i}, i=1,2, \ldots, N$, replaced by the functions

$$
[a, b] \ni t \longmapsto\left|R_{i}(t)\right| \operatorname{sign}(t-\tau), \quad i=1,2, \ldots, N .
$$

Applying Proposition 4.4 to the operator $A: C_{\tau}\left([a, b], \mathbb{R}^{n}\right) \rightarrow C_{\tau}\left([a, b], \mathbb{R}^{n}\right)$ given by the formula (4.24), we conclude that its spectral radius admits the estimate

$$
r(A)<1 \text {. }
$$


In view of (4.28), inequality (4.34) implies that $r(B)<1$. Now, similarly to Theorem 4.1, it remains to apply Lemma 4.1.

\section{Proofs of the results of Section 3}

Let us put $N:=n^{2}$ and, for any $v=1,2, \ldots, N$,

$$
k_{v}:=\left\lfloor\frac{v-1}{n}\right\rfloor+1
$$

and

$$
j_{v}:=v-n\left\lfloor\frac{v-1}{n}\right\rfloor,
$$

where the symbol $\lfloor\cdot\rfloor$ stands for the integer part of a number. Furthermore, define the functions $\omega_{v}:[a, b] \rightarrow[a, b]$ and $R_{v}:[a, b] \rightarrow \mathrm{GL}_{n}(\mathbb{R}), v=1,2, \ldots, n^{2}$, by setting

$$
\omega_{v}(t):=\omega_{k_{v} j_{v}}(t)
$$

and

$$
R_{v}(t):=r_{k_{v} j_{v}}(t) \Pi_{v},
$$

where $v=1,2, \ldots, n^{2}, t \in[a, b]$, and $\Pi_{v}$ is the $n$-dimensional matrix the only non-zero element of which is equal to 1 and located at row $k_{v}$ and column $j_{v}, v=$ $1,2, \ldots, n^{2}$; in other words,

$$
\Pi_{v}=\left(\begin{array}{cccc}
\delta_{1, k_{v}} \delta_{1, j_{v}} & \delta_{1, k_{v}} \delta_{2, j_{v}} & \ldots & \delta_{1, k_{v}} \delta_{n, j_{v}} \\
\delta_{2, k_{v}} \delta_{1, j_{v}} & \delta_{2, k_{v}} \delta_{2, j_{v}} & \ldots & \delta_{2, k_{v}} \delta_{n, j_{v}} \\
\ldots \ldots \ldots \ldots \ldots \ldots \ldots \ldots \ldots \ldots \ldots \ldots \ldots & \ldots \ldots \ldots \ldots \\
\delta_{n, k_{v}} \delta_{1, j_{v}} & \delta_{n, k_{v}} \delta_{2, j_{v}} & \ldots & \delta_{n, k_{v}} \delta_{n, j_{v}}
\end{array}\right),
$$

where $\delta_{k, j}, k, j=1,2, \ldots, n$, is the Kronecker symbol.

5.1. Lemmata. Let us establish several auxiliary statements.

Lemma 5.1. For any $v$ from 1 to $n^{2}$, the equality

$$
\left(k_{v}-1\right) n+j_{v}=v
$$

is true. Furthermore,

$$
\left\{k_{v} \mid v=1,2, \ldots, n^{2}\right\}=\{1,2, \ldots, n\}
$$

and

$$
\left\{j_{v} \mid v=1,2, \ldots, n^{2}\right\}=\{1,2, \ldots, n\}
$$


Proof. Equality (5.6) is obvious from formulae (5.1) and (5.2). To establish (5.7) and (5.8), one should note that (5.1) can be rewritten as the system of equalities

$$
\begin{aligned}
& k_{1}=k_{2}=\cdots=k_{n}=1, \\
& k_{n+1}=k_{n+2}=\cdots=k_{2 n}=2 \text {, } \\
& k_{2 n+1}=k_{2 n+2}=\cdots=k_{3 n}=3 \text {, } \\
& k_{(n-1) n+1}=k_{(n-1) n+2}=\cdots=k_{n^{2}}=n \text {, }
\end{aligned}
$$

and, similarly, relations (5.2) mean that

$$
\begin{gathered}
j_{1}=j_{n+1}=j_{2 n+1}=\cdots=j_{(n-1) n+1}=1, \\
j_{2}=j_{n+2}=j_{2 n+2}=\cdots=j_{(n-1) n+2}=2, \\
j_{3}=j_{n+3}=j_{2 n+3}=\cdots=j_{(n-1) n+3}=3, \\
\cdots \cdots \cdots \cdots \cdots \cdots \cdots \cdots \cdots \cdots \cdots+j_{n^{2}}=n .
\end{gathered}
$$

Relations (5.9) and (5.10) lead us to the required equalities (5.7) and (5.8).

Lemma 5.2. A number $v \in\left\{1,2, \ldots, n^{2}\right\}$ satisfies the condition $k_{v}=k$, where $k \in\{1,2, \ldots, n\}$, if, and only if it satisfies the inequality

$$
1 \leq v-(k-1) n \leq n,
$$

in which case

$$
j_{v}=v-(k-1) n+1 .
$$

Proof. Taking (5.9) and (5.10) into account, we conclude that condition (5.14) is necessary and sufficient for the fulfilment of the equality $k=k_{v}$. Equality (5.12) is an immediate consequence of relation (5.6).

For all $k$ from 1 to $n$, put

$$
e_{k}:=\operatorname{col}(\underbrace{0,0, \ldots, 0}_{k-1}, 1,0, \ldots, 0) .
$$

Lemma 5.3. Let $\left\{m_{k j} \mid k, j=1,2, \ldots, n\right\} \subset \mathbb{R}$ be arbitrary constants and $v=$ $\left(v_{j}\right)_{j=1}^{n}$ be an arbitrary vector. Then the equality

$$
\sum_{v=1}^{n^{2}} m_{k_{v} j_{v}} e_{k}^{*} \Pi_{v} v=\sum_{j=1}^{n} m_{k j} v_{j}
$$

is true for any $k$ from 1 to $n$.

Recall that the $n \times n$ matrices $\Pi_{v}, v=1,2, \ldots, n^{2}$, appearing in equality (5.13) are defined by formula (5.5). The asterisk in (5.13) and similar formulae below marks the transposed vector. 
Proof of Lemma 5.3. In view of (5.5), for any $v$ from 1 to $n^{2}$, we have

$$
e_{k}^{*} \Pi_{v} v=\sum_{j=1}^{n} \delta_{k, k_{v}} \delta_{j, j_{v}}=\delta_{k, k_{v}} v_{j_{v}}= \begin{cases}v_{j_{v}} & \text { if } k=k_{v}, \\ 0 & \text { in the contrary case. }\end{cases}
$$

By virtue of Lemma 5.2, an integer $v$ satisfies the equality $k_{v}=k$ if, and only if it is represented in the form

$$
v=(k-1) n+p
$$

where $1 \leq p \leq n$. Therefore, for any $v$ and $k$, we have

$$
m_{k_{v} j_{v}} e_{k}^{*} \Pi_{v} v= \begin{cases}m_{k, p+1} v_{p+1} & \text { if } v \text { satisfies (5.14) and } 1 \leq p \leq n, \\ 0 & \text { in the contrary case. }\end{cases}
$$

The last relation yields the equality

$$
\sum_{v=1}^{n^{2}} m_{k_{v} j_{v}} e_{k}^{*} \Pi_{v} v=\sum_{p=1}^{n} m_{k, p} v_{p}
$$

which coincides with (5.13).

The following lemma establishes some relations for the functions $R_{v}:[a, b] \rightarrow$ $\mathrm{GL}_{n}(\mathbb{R}), v=1,2, \ldots, n^{2}$, defined by formula (5.4).

Lemma 5.4. Let $v=\left(v_{j}\right)_{j=1}^{n} \in \mathbb{R}^{n}$ be an arbitrary vector and $\phi_{v}, v=1,2, \ldots, n^{2}$, be arbitrary constants. Then the equalities

$$
e_{k}^{*} \sum_{v=1}^{n^{2}} \phi_{v} R_{v}(t) v=\sum_{j=1}^{n} \phi_{(k-1) n+j} r_{k j}(t) v_{j}
$$

and

$$
e_{k}^{*} \sum_{v=1}^{n^{2}} \phi_{v}\left|R_{v}(t)\right| v=\sum_{j=1}^{n} \phi_{(k-1) n+j}\left|r_{k j}(t)\right| v_{j}
$$

are true for any $k$ from 1 to $n$ and almost every $t \in[a, b]$.

Proof. Application of Lemma 5.3 with

$$
m_{k j}:=r_{k j}(t) \phi_{(k-1) n+j},
$$

where $t \in[a, b]$ and $k, j=1,2, \ldots, n$, yields

$$
\sum_{v=1}^{n^{2}} \phi_{\left(k_{v}-1\right) n+j_{v}} r_{k_{v} j_{v}}(t) e_{k}^{*} \Pi_{v} v=\sum_{j=1}^{n} \phi_{(k-1) n+j} r_{k j}(t) v_{j} .
$$

Taking equality (5.6) into account, one can rewrite (5.18) in the form

$$
\sum_{v=1}^{n^{2}} \phi_{v} r_{k_{v} j_{v}}(t) e_{k}^{*} \Pi_{v} v=\sum_{j=1}^{n} \phi_{(k-1) n+j} r_{k j}(t) v_{j}
$$


It follows from (5.4) that $r_{k_{v} j_{v}}(t) \Pi_{v}=R_{v}(t)$ for any $v$ and $t$, and, hence (5.19) means that (5.16) is satisfied. Relation (5.17) is proved similarly.

Lemma 5.5. Let $v^{(v)}=\left(v_{j}^{(v)}\right)_{j=1}^{n}, v=1,2, \ldots, n^{2}$, be arbitrary $n$-dimensional vectors. Then the equalities

$$
e_{k}^{*} \sum_{v=1}^{n^{2}} R_{v}(t) v^{(v)}=\sum_{j=1}^{n} v_{j}^{((k-1) n+j)} r_{k j}(t)
$$

and

$$
e_{k}^{*} \sum_{v=1}^{n^{2}}\left|R_{v}(t)\right| v^{(v)}=\sum_{j=1}^{n} v_{j}^{((k-1) n+j)}\left|r_{k j}(t)\right|
$$

are true for any $k$ from 1 to $n$ and almost every $t \in[a, b]$.

Proof. In view of the equality

$$
v^{(v)}=\sum_{i=1}^{n} v_{i}^{(v)} e_{i},
$$

for almost every $t \in[a, b]$ and all $k=1,2, \ldots, n$, we have

$$
\begin{aligned}
e_{k}^{*} \sum_{v=1}^{n^{2}} R_{v}(t) v^{(v)} & =e_{k}^{*} \sum_{v=1}^{n^{2}} R_{v}(t) \sum_{i=1}^{n} v_{i}^{(v)} e_{i} \\
& =\sum_{i=1}^{n}\left(e_{k}^{*} \sum_{v=1}^{n^{2}} v_{i}^{(v)} R_{v}(t) e_{i}\right) .
\end{aligned}
$$

In view of Lemma 5.4 applied with $v:=e_{i}$ and $\phi_{v}:=v_{i}^{(v)}, v=1,2, \ldots, n^{2}$, equality (5.22) yields

$$
\begin{aligned}
e_{k}^{*} \sum_{v=1}^{n^{2}} R_{v}(t) v^{(v)} & =\sum_{i=1}^{n}\left(\sum_{j=1}^{n} v_{i}^{((k-1) n+j)} r_{k j}(t) \delta_{i, j}\right) \\
& =\sum_{j=1}^{n}\left(\sum_{i=1}^{n} v_{i}^{((k-1) n+j)} \delta_{i, j}\right) r_{k j}(t) \\
& =\sum_{j=1}^{n} v_{j}^{((k-1) n+j)} r_{k j}(t)
\end{aligned}
$$

for all $k$ from 1 to $n$ and almost every $t \in[a, b]$, which proves (5.20). Relation (5.21) is established by analogy.

Lemma 5.6. The equality

$$
\omega_{(k-1) n+j}(t)=\omega_{k j}(t)
$$


is satisfied for almost every $t \in[a, b]$ and all $k$ and $j$ from 1 to $n$.

Proof. Let $\{k, j\} \subset\{1,2, \ldots, n\}$ be arbitrary. Then, obviously, $k=k_{v}$ and $j=j_{v}$ for $v:=(k-1) n+j$. Applying the equality (5.6) of Lemma 5.1 and using the definition (5.3) of the functions $\omega_{v}, v=1,2, \ldots, n^{2}$, we obtain that

$$
\omega_{\left(k_{v}-1\right) n+j_{v}}(t)=\omega_{v}(t)=\omega_{k_{v} j_{v}}(t)
$$

for almost every $t \in[a, b]$. This yields the required property (5.23).

Lemma 5.7. Problem (1.2), (1.3) is equivalent to problem (4.12), (4.13), where $N=n^{2}$ and $\omega_{v}:[a, b] \rightarrow[a, b]$ and $R_{v}:[a, b] \rightarrow \mathrm{GL}_{n}(\mathbb{R}), v=1,2, \ldots, N$, are defined by formulae (5.3) and (5.4), respectively.

Proof. Let $u=\left(u_{k}\right)_{k=1}^{n}:[a, b] \rightarrow \mathbb{R}^{n}$ be an arbitrary continuous vector-function. Applying formula (5.20) of Lemma 5.5 with $v^{(v)}:=u\left(\omega_{v}(t)\right), v=1,2, \ldots, n^{2}$, for any $k$ from 1 to $n$, we obtain

$$
e_{k}^{*} \sum_{v=1}^{n^{2}} R_{v}(t) u\left(\omega_{v}(t)\right)=\sum_{j=1}^{n} u_{j}\left(\omega_{(k-1) n+j}(t)\right) r_{k j}(t)
$$

and therefore, by Lemma 5.6,

$$
e_{k}^{*} \sum_{v=1}^{n^{2}} R_{v}(t) u\left(\omega_{v}(t)\right)=\sum_{j=1}^{n} r_{k j}(t) u_{j}\left(\omega_{k j}(t)\right)
$$

for almost every $t$ from $[a, b]$. Comparing the right-hand side member of (5.24) with that of (1.2) and taking the arbitrariness of $u$ and $k$ into account, we arrive at the desired conclusion.

Lemma 5.8. Assume that the functions $r_{k j}:[a, b] \rightarrow \mathbb{R}, k, j=1,2, \ldots, n$, satisfy relations (3.4) for almost every $t \in[a, b]$ and all $k, j=1,2, \ldots, n$. Then the corresponding functions $R_{v}:[a, b] \rightarrow \mathrm{GL}_{n}(\mathbb{R}), v=1,2, \ldots, n^{2}$, given by formula (5.4) satisfy condition (4.3) with respect to matrix (4.2).

Proof. Indeed, according to Lemma 4.3, condition (4.3) is satisfied if, and only if the components $r_{k j}^{(v)}:[a, b] \rightarrow \mathbb{R}, k, j=1,2, \ldots, n$, of the matrices $R_{v}:[a, b] \rightarrow$ $\mathrm{GL}_{n}(\mathbb{R}), v=1,2, \ldots, n^{2}$, satisfy inequalities (4.19) almost everywhere on $[a, b]$. By virtue of (5.4), for any $v=1,2, \ldots, n^{2}$ and almost every $t \in[a, b]$, we have

$$
r_{k j}^{(v)}(t)= \begin{cases}r_{k_{v} j_{v}}(t) & \text { if } k=k_{v} \text { and } j=j_{v}, \\ 0 & \text { in the contrary case. }\end{cases}
$$

In view of (5.25), the fulfilment of all the inequalities (4.19) means that

$$
\sigma_{k_{v}} \sigma_{j_{v}} r_{k_{v} j_{v}}(t) \operatorname{sign}(t-\tau) \geq 0
$$

for all $v=1,2, \ldots, n^{2}$ and almost every $t \in[a, b]$. However, it follows from Lemma 5.1 that equalities (5.7) and (5.8) are true and, therefore, the fulfilment of 
conditions (3.4) for all $k, j=1,2, \ldots, n$ and almost every $t \in[a, b]$ ensures that relation (5.26) is satisfied for all $v=1,2, \ldots, n^{2}$ and almost every $t \in[a, b]$. We have thus shown that functions (5.4) satisfy condition (4.3).

5.2. Proof of Theorem 3.1. Let us show that, under the conditions assumed, the required assertion for the Cauchy problem (1.2), (1.3) follows from Theorem 4.2 applied to the corresponding problem (4.12), (4.13).

Case 1: Condition (3.1a) holds. Assumption (3.1a) ensures that the functions $\omega_{v}$ : $[a, b] \rightarrow[a, b]$ and $R_{v}:[a, b] \rightarrow \mathrm{GL}_{n}(\mathbb{R}), v=1,2, \ldots, n^{2}$, given by formulae (5.3) and (5.4) satisfy condition (4.23) of Theorem 4.2 with

$$
g:=\operatorname{col}\left(\gamma_{1}, \gamma_{2}, \ldots, \gamma_{n}\right)
$$

and

$$
\gamma:=\frac{1}{\alpha} \max _{k=1,2, \ldots, n} \operatorname{vrai}_{t \in[a, b] \backslash\{\tau\}} \frac{1}{\gamma_{k}|t-\tau|^{\alpha-1}} \sum_{j=1}^{n} \gamma_{j}\left|r_{k j}(t)\right|\left|\omega_{j}(t)-\tau\right|^{\alpha} .
$$

Indeed, let us put $v_{j}=\gamma_{j}, j=1,2, \ldots, n$. Applying equality (5.17) of Lemma 5.4 and taking formulae (5.3) and (5.12) into account, we obtain that

$$
e_{k}^{*} \sum_{v=1}^{n^{2}} \omega_{v}(t)\left|R_{v}(t)\right| g=\sum_{j=1}^{n} \omega_{(k-1) n+j}(t)\left|r_{k j}(t)\right| \gamma_{j}
$$

for almost every $t \in[a, b]$ and all $k$ from 1 to $n$. By virtue of Lemma 5.6, relation (5.29) can be brought to the form

$$
e_{k}^{*} \sum_{v=1}^{n^{2}} \omega_{v}(t)\left|R_{v}(t)\right| g=\sum_{j=1}^{n} \omega_{k j}(t)\left|r_{k j}(t)\right| \gamma_{j}
$$

Therefore, property (4.23) in our case is equivalent to the fulfilment of the inequality

$$
\sum_{j=1}^{n}\left|r_{k j}(t)\right| \gamma_{j}\left|\omega_{k j}(t)-\tau\right|^{\alpha} \leq \gamma \alpha \gamma_{k}|t-\tau|^{\alpha-1}
$$

for almost every $t \in[a, b]$ and all $k$ from 1 to $n$. However, this condition is satisfied in view of (3.1a) and (5.28).

Case 2: Condition (3.1b) holds. In this case, condition (4.21) of Theorem 4.2 is satisfied. Indeed, let us define the vector $g$ by formula (5.27), where $\gamma_{1}, \gamma_{2}, \ldots, \gamma_{n}$ are the positive constants appearing in (3.1b). Then condition (4.21) is equivalent to the system of relations

$$
\sum_{v=1}^{N} \int_{\tau}^{t} e_{k}^{*}\left|R_{v}(s)\right| g\left|\omega_{v}(s)-\tau\right|^{\alpha} d s \cdot \operatorname{sign}(t-\tau) \leq \gamma|t-\tau|^{\alpha} \gamma_{k}
$$

valid for all $k=1,2, \ldots, n$ and $t \in[a, b]$. 
Applying equality (5.17) of Lemma 5.4 with $v_{j}:=\gamma_{j}, j=1,2, \ldots, n$, and $\phi_{v}:=$ $\left|\omega_{v}(s)-\tau\right|^{\alpha}, v=1,2, \ldots, n^{2}$, and using Lemma 5.6, we show that the equalities

$$
\begin{aligned}
\sum_{v=1}^{N} e_{k}^{*}\left|\omega_{v}(s)-\tau\right|^{\alpha}\left|R_{v}(s)\right| g & =\sum_{j=1}^{n}\left|\omega_{(k-1) n+j}(s)-\tau\right|^{\alpha}\left|r_{k j}(s)\right| \gamma_{j} \\
& =\sum_{j=1}^{n}\left|\omega_{k j}(s)-\tau\right|^{\alpha}\left|r_{k j}(s)\right| \gamma_{j}
\end{aligned}
$$

are satisfied for any $k=1,2, \ldots, n$ and almost every $s \in[a, b]$. Therefore, relation (5.30) in our case means that, for all $k=1,2, \ldots, n$ and $t \in[a, b]$, the inequality

$$
\sum_{j=1}^{n} \gamma_{j} \int_{\tau}^{t}\left|r_{k j}(s)\right|\left|\omega_{k j}(s)-\tau\right|^{\alpha} d s \cdot \operatorname{sign}(t-\tau) \leq \gamma \gamma_{k}|t-\tau|^{\alpha} .
$$

is true. Setting now

$$
\gamma:=\max _{k=1,2, \ldots, n} \sup _{t \in[a, b] \backslash\{\tau\}} \frac{\operatorname{sign}(t-\tau)}{\gamma_{k}|t-\tau|^{\alpha}} \sum_{j=1}^{n} \gamma_{j} \int_{\tau}^{t}\left|r_{k j}(s)\right|\left|\omega_{k j}(s)-\tau\right|^{\alpha} d s
$$

and taking assumption (3.1b) into account, we conclude that (5.31) is satisfied for all $t \in[a, b]$ and $k$ from 1 to $n$. As we have already seen above, this means that relation (4.21) is true.

Case 3: Condition (3.1c) holds. Let us show that, in this case, condition (4.22) is satisfied with the vector $g$ defined by formula (5.27) and

$$
\begin{aligned}
\gamma:=\frac{1}{\alpha} \max _{k=1,2, \ldots, n, n} \underset{t \in[a, b] \backslash\{\tau\}}{\operatorname{vrai} \max } \sum_{l=1}^{n} \frac{\operatorname{sign}\left(\omega_{k l}(t)-\tau\right)}{\gamma_{k} \mid t}-\left.\tau\right|^{\alpha-1}\left|r_{k l}(t)\right| \\
\quad \times \sum_{j=1}^{n} \gamma_{j} \int_{\tau}^{\omega_{k l}(t)}\left|r_{l j}(s) \| \omega_{l j}(s)-\tau\right|^{\alpha} d s .
\end{aligned}
$$

Indeed, in the case indicated, condition (4.22) for functions (5.3) and (5.4) means that, for all $k=1,2, \ldots, n$ and almost every $t \in[a, b]$,

$$
\begin{aligned}
\sum_{v=1}^{N} \operatorname{sign}\left(\omega_{v}(t)-\tau\right) e_{k}^{*}\left|R_{v}(t)\right| \sum_{i=1}^{N} \int_{\tau}^{\omega_{v}(t)}\left|R_{i}(s)\right| g\left|\omega_{i}(s)-\tau\right|^{\alpha} d s & \\
& \leq \gamma \alpha|t-\tau|^{\alpha-1} \gamma_{k}
\end{aligned}
$$

or, which is the same,

$$
\sum_{v=1}^{n^{2}} e_{k}^{*}\left|R_{v}(t)\right| v^{(v)}(t) \leq \gamma \alpha|t-\tau|^{\alpha-1} \gamma_{k}
$$


where

$$
v^{(v)}(t):=\sum_{i=1}^{n^{2}} \int_{\tau}^{\omega_{v}(t)}\left|R_{i}(s)\right| g\left|\omega_{i}(s)-\tau\right|^{\alpha} d s \operatorname{sign}\left(\omega_{v}(t)-\tau\right)
$$

for any $v=1,2, \ldots, n^{2}$. Applying formula (5.21) of Lemma 5.5, we obtain

$$
\begin{aligned}
& e_{k}^{*} \sum_{v=1}^{n^{2}}\left|R_{v}(t)\right| v^{(v)}(t)=\sum_{j=1}^{n} v_{j}^{((k-1) n+j)}(t)\left|r_{k j}(t)\right| \\
& \quad=\sum_{j=1}^{n}\left|r_{k j}(t)\right| e_{j}^{*}\left(\sum_{i=1}^{n^{2}} \int_{\tau}^{\omega_{(k-1) n+j}(t)}\left|R_{i}(s)\right| g\left|\omega_{i}(s)-\tau\right|^{\alpha} d s\right) \operatorname{sign}\left(\omega_{(k-1) n+j}(t)-\tau\right),
\end{aligned}
$$

whence, by Lemma 5.6, it follows that

$$
\begin{aligned}
e_{k}^{*} \sum_{v=1}^{n^{2}} \mid & R_{v}(t) \mid v^{(v)}(t) \\
\quad & =\sum_{j=1}^{n}\left|r_{k j}(t)\right| \int_{\tau}^{\omega_{k j}(t)}\left(e_{j}^{*} \sum_{i=1}^{n^{2}}\left|R_{i}(s)\right| g\left|\omega_{i}(s)-\tau\right|^{\alpha}\right) d s \operatorname{sign}\left(\omega_{k j}(t)-\tau\right)
\end{aligned}
$$

for almost every $t$ from $[a, b]$. On the other hand, by virtue of formula (5.27) and Lemmata 5.4 and 5.6, the equality

$$
\begin{aligned}
e_{j}^{*} \sum_{i=1}^{n^{2}}\left|R_{i}(s)\right| g\left|\omega_{i}(s)-\tau\right|^{\alpha} & =\sum_{l=1}^{n} \gamma_{l}\left|r_{j l}(s)\right|\left|\omega_{(j-1) n+l}(s)-\tau\right|^{\alpha} \\
& =\sum_{l=1}^{n} \gamma_{l}\left|r_{j l}(s)\right|\left|\omega_{j l}(s)-\tau\right|^{\alpha}
\end{aligned}
$$

is true for almost every $s \in[a, b]$ and all $j=1,2, \ldots, n$. Using (5.36) and (5.35), we obtain that

$$
\begin{aligned}
& e_{k}^{*} \sum_{v=1}^{n^{2}}\left|R_{v}(t)\right| v^{(v)}(t) \\
& \quad=\sum_{j=1}^{n}\left|r_{k j}(t)\right|\left(\sum_{l=1}^{n} \gamma_{l} \int_{\tau}^{\omega_{k j}(t)}\left|r_{j l}(s)\right|\left|\omega_{j l}(s)-\tau\right|^{\alpha} d s\right) \operatorname{sign}\left(\omega_{k j}(t)-\tau\right)
\end{aligned}
$$


for all $k=1,2, \ldots, n$ and almost every $t \in[a, b]$. Substituting (5.37) into (5.34), we bring the latter relation to the form

$$
\begin{aligned}
\sum_{j=1}^{n}\left|r_{k j}(t)\right|\left(\sum_{l=1}^{n} \gamma_{l} \int_{\tau}^{\omega_{k j}(t)}\left|r_{j l}(s)\right|\left|\omega_{j l}(s)-\tau\right|^{\alpha} d s\right) \operatorname{sign}\left(\omega_{k j}(t)-\tau\right) & \\
& \leq \gamma \alpha|t-\tau|^{\alpha-1} \gamma_{k} .
\end{aligned}
$$

Taking (5.32) into account, we conclude that (5.38) is satisfied for all $k=1,2, \ldots, n$ and almost every $t \in[a, b]$ in view of condition (3.1c).

We have thus shown that, under the conditions assumed, Theorem 4.2 can be applied to problem (4.12), (4.13) with $N=n^{2}$ and the functions $\omega_{v}$ and $R_{v}, v=$ $1,2, \ldots, n^{2}$, given by formulae (5.3) and (5.4). Using the theorem mentioned and taking Lemma 5.7 into account, we arrive at the desired assertion on the unique solvability of the original Cauchy problem (1.2), (1.3).

5.3. Proof of Theorem 3.2. We shall show that the assertion of Theorem 3.2 for the Cauchy problem (1.2), (1.3) can be obtained from Theorem 4.1 applied to the corresponding problem (4.12), (4.13).

Indeed, by virtue of assumption (3.4) and Lemma 5.8, the functions $\omega_{v}:[a, b] \rightarrow$ $[a, b]$ and $R_{v}:[a, b] \rightarrow \mathrm{GL}_{n}(\mathbb{R}), v=1,2, \ldots, n^{2}$, defined by formulae (5.3) and (5.4), satisfy condition (4.3) with $\Sigma$ given by (4.2). Therefore, it is sufficient to show that, under our assumptions on $r_{k j}$ and $\omega_{k j}, k, j=1,2, \ldots, n$, some of conditions (4.8), (4.10), and (4.11) are satisfied.

Let us put

$$
g:=\operatorname{col}\left(\sigma_{1} \gamma_{1}, \sigma_{2} \gamma_{2}, \ldots, \sigma_{n} \gamma_{n}\right),
$$

where $\gamma_{1}, \gamma_{2}, \ldots, \gamma_{n}$ are the positive constants appearing in conditions (3.5a), (3.5b), and (3.5c). Obviously, vector (5.39) possesses property (4.7).

Consider the following three cases.

Case 1: Condition (3.5a) holds. Let us prove that, under assumption (3.5a), the functions $\omega_{v}$ and $R_{v}, v=1,2, \ldots, n^{2}$, in the corresponding system (4.13) satisfy condition (4.11) of Theorem 4.1.

Indeed, applying formula (5.16) of Lemma 5.4, we show that condition (4.11) is equivalent to the fulfilment of the relation

$$
\begin{aligned}
\sigma_{k} \sum_{j=1}^{n} \sigma_{j} \gamma_{j} r_{k j}(t)\left|\omega_{(k-1) n+j}(t)-\tau\right|^{\alpha} \operatorname{sign}(t-\tau) & \leq \sigma_{k} \gamma \sigma_{k} \gamma_{k} \alpha|t-\tau|^{\alpha} \\
& =\gamma \gamma_{k} \alpha|t-\tau|^{\alpha}
\end{aligned}
$$


for all $k=1,2, \ldots, n$ and almost every $t \in[a, b]$. By Lemma 5.6, relation (5.40) means that

$$
\sigma_{k} \sum_{j=1}^{n} \sigma_{j} \gamma_{j} r_{k j}(t)\left|\omega_{k j}(t)-\tau\right|^{\alpha} \operatorname{sign}(t-\tau) \leq \gamma_{k} \gamma \alpha|t-\tau|^{\alpha}
$$

for all $k=1,2, \ldots, n$ and almost every $t \in[a, b]$. However, the existence of a constant $\gamma \in[0,1)$ such that $(5.41)$ holds for all $k=1,2, \ldots, n$ and almost every $t \in[a, b]$ is a consequence of assumption ( $3.5 \mathrm{a}$ ) because one can put

$$
\gamma:=\max _{k=1,2, \ldots, n, n} \underset{v r a i \max }{\operatorname{vrab} \max _{t \in[\tau\}}} \frac{\sigma_{k} \operatorname{sign}(t-\tau)}{\alpha \gamma_{k}|t-\tau|^{\alpha-1}} \sum_{j=1}^{n} \sigma_{j} \gamma_{j} r_{k j}(t)\left|\omega_{k j}(t)-\tau\right|^{\alpha} .
$$

Case 2: Condition (3.5b) holds. Let us show that functions (5.3) and (5.4) in this case satisfy condition (4.8).

It follows from Lemma 5.4 that condition (4.8) with $g$ given by formula (5.39) means that

$$
\sigma_{k} \sum_{j=1}^{n} \int_{\tau}^{t} \sigma_{j} \gamma_{j}\left|\omega_{(k-1) n+j}(s)-\tau\right|^{\alpha} r_{k j}(s) d s \leq \gamma|t-\tau|^{\alpha} \gamma_{k},
$$

or, equivalently (see Lemma 5.6)

$$
\sigma_{k} \sum_{j=1}^{n} \sigma_{j} \gamma_{j} \int_{\tau}^{t}\left|\omega_{k j}(s)-\tau\right|^{\alpha} r_{k j}(s) d s \leq \gamma_{k} \gamma|t-\tau|^{\alpha}
$$

for all $t \in[a, b]$ and $k$ from 1 to $n$. A constant $\gamma \in[0,1)$ such that (5.42) holds for all $k$ and $t$ exists due to assumption (3.5b).

Case 3: Condition (3.5c) holds. Assumption (3.5c) ensures that the functions $\omega_{v}$ and $R_{v}, v=1,2, \ldots, n^{2}$, in the corresponding system (4.13) satisfy condition (4.10) of Theorem 4.1 .

Indeed, let us define the vector $g$ by formula (5.39). Condition (4.10) for functions (5.3) and (5.4) in our case means that, for all $k=1,2, \ldots, n$ and almost every $t \in[a, b]$,

$$
\begin{aligned}
\sigma_{k} \operatorname{sign}(t-\tau) \sum_{v=1}^{N} e_{k}^{*} R_{v}(t) \sum_{i=1}^{N} \int_{\tau}^{\omega_{v}(t)} R_{i}(s) g\left|\omega_{i}(s)-\tau\right|^{\alpha} d s & \\
& \leq \gamma \gamma_{k} \alpha|t-\tau|^{\alpha-1}
\end{aligned}
$$

or, which is the same,

$$
\sigma_{k} \operatorname{sign}(t-\tau) \sum_{v=1}^{n^{2}} e_{k}^{*} R_{v}(t) w^{(v)}(t) \leq \gamma \gamma_{k} \alpha|t-\tau|^{\alpha-1},
$$


where

$$
w^{(v)}(t):=\sum_{i=1}^{n^{2}} \int_{\tau}^{\omega_{v}(t)} R_{i}(s) g\left|\omega_{i}(s)-\tau\right|^{\alpha} d s
$$

for any $v=1,2, \ldots, n^{2}$. Formula (5.20) of Lemma 5.5 implies that, for almost every $t \in[a, b]$ and all $k=1,2, \ldots, n$,

$$
\begin{aligned}
e_{k}^{*} \sum_{v=1}^{n^{2}} R_{v}(t) w^{(v)}(t)=\sum_{j=1}^{n} w_{j}^{((k-1) n+j)}(t) r_{k j}(t) & \\
& =\sum_{j=1}^{n} r_{k j}(t) e_{j}^{*}\left(\sum_{i=1}^{n^{2}} \int_{\tau}^{\omega_{(k-1) n+j}(t)} R_{i}(s) g\left|\omega_{i}(s)-\tau\right|^{\alpha} d s\right),
\end{aligned}
$$

whence, by Lemma 5.6,

$$
e_{k}^{*} \sum_{v=1}^{n^{2}} R_{v}(t) w^{(v)}(t)=\sum_{j=1}^{n} r_{k j}(t) \int_{\tau}^{\omega_{k j}(t)}\left(e_{j}^{*} \sum_{i=1}^{n^{2}} R_{i}(s) g\left|\omega_{i}(s)-\tau\right|^{\alpha}\right) d s .
$$

However, it follows from Lemmata 5.4 and 5.6 and formula (5.39) that the equality

$$
\begin{aligned}
e_{j}^{*} \sum_{i=1}^{n^{2}} R_{i}(s) g\left|\omega_{i}(s)-\tau\right|^{\alpha} & =\sum_{l=1}^{n} \sigma_{l} \gamma_{l} r_{j l}(s)\left|\omega_{(j-1) n+l}(s)-\tau\right|^{\alpha} \\
& =\sum_{l=1}^{n} \sigma_{l} \gamma_{l} r_{j l}(s)\left|\omega_{j l}(s)-\tau\right|^{\alpha}
\end{aligned}
$$

is true for almost every $s \in[a, b]$ and all $j=1,2, \ldots, n$, and, therefore, equality (5.45) yields

$$
e_{k}^{*} \sum_{v=1}^{n^{2}} R_{v}(t) w^{(v)}(t)=\sum_{j=1}^{n} r_{k j}(t) \int_{\tau}^{\omega_{k j}(t)}\left(\sum_{l=1}^{n} \sigma_{l} \gamma_{l} r_{j l}(s)\left|\omega_{j l}(s)-\tau\right|^{\alpha}\right) d s .
$$

In view of (5.46), relation (5.44) can be rewritten as

$$
\begin{aligned}
\sigma_{k} \operatorname{sign}(t-\tau) \sum_{j=1}^{n} r_{k j}(t) \int_{\tau}^{\omega_{k j}(t)}\left(\sum_{l=1}^{n} \sigma_{l} \gamma_{l} r_{j l}(s)\left|\omega_{j l}(s)-\tau\right|^{\alpha}\right) d s & \\
& \leq \gamma \gamma_{k} \alpha|t-\tau|^{\alpha-1} .
\end{aligned}
$$

It remains to note that the existence of a constant $\gamma \in[0,1)$ such that $(5.47)$ is true for almost every $t \in[a, b]$ and all $k=1,2, \ldots, n$ is guaranteed by condition (3.5c).

Thus, in each of the three cases considered above, the conditions of imposed on problem (1.2), (1.3) in Theorem 3.2 guarantee that the corresponding problem (4.12), 
(4.13) satisfies all the assumptions of Theorem 4.1. It therefore remains to use Theorem 4.1 and Lemma 5.7.

\section{REFERENCES}

[1] Azbelev, N., Maksimov, V., and Rakhmatullina, L.: Introduction to the Theory of Linear Functional Differential Equations, vol. 3 of Advanced Series in Mathematical Science and Engineering, World Federation Publishers Company, Atlanta, GA, 1995.

[2] Dilnaya, N. and Rontó, A.: Multistage iterations and solvability of linear Cauchy problems, Miskolc Math. Notes, 4 (2003), No. 2, 89-102.

[3] Dilnaya, N. Z. and Ronto, A. N.: Some new solvability conditions of the Cauchy problem for systems of linear functional differential equations, Ukrain. Math. J., 56 (2004), No. 7, 867-884.

[4] Hakl, R., Lomtatidze, A., and Šremr, J.: Some Boundary Value Problems for First Order Scalar Functional Differential Equations, Masaryk University, Brno, 2002.

[5] Krasnoselskit, M. A., Vainikko, G. M., Zabreiko, P. P., Rutitskit, Y. B., and Stetsenko, V. Y.: Approximate Solution of Operator Equations, Noordhoff, Groningen, 1972.

[6] Ronto, A. N.: Exact solvability conditions of the Cauchy problem for systems of linear firstorder functional differential equations determined by $\left(\sigma_{1}, \sigma_{2}, \ldots, \sigma_{n} ; \tau\right)$-positive operators, Ukrain. Math. J., 55 (2003), No. 11, 1853-1884.

\section{Author's Address}

\section{Andrei Rontó:}

Institute of Mathematics, National Academy of Sciences of Ukraine, 3 Tereschenkovskaya St., 01601 KiEv, Ukraine

Current address: Mathematical Institute, Academy of Sciences of the Czech Republic, Žižkova 22, CZ-61662 Brno, Czech Republic

E-mail address: ronto@ipm.cz 